ISSN: $1130-3743$

DOI: http://dx.doi.org/10.14201/teoredu2014261183204

\title{
IMPACTO DE LAS TECNOLOGÍAS DIGITALES EN LA DESCENTRALIZACIÓN DEL SISTEMA ESCOLAR
}

\author{
Impact of digital technologies on decentralization \\ of the school system
}

\section{Impact des technologies numériques sur la décentralisation du système scolaire}

\author{
Ángel SAn MarTín Alonso y José Peirats Chacón \\ Universitat de València. Facultat de Filosofia i Ciències de l'Educació. \\ Departament de Didàctica i Organització Escolar. Avda. Blasco Ibáñez, 30. \\ 46010 València. asanmart@uv.es, jose.peirats@uv.es
}

Fecha de recepción: marzo de 2014

Fecha de aceptación: junio de 2014

Biblid [(1130-3743) 26, 2-2014, 183-204]

\section{RESUMEN}

Nuestro objetivo es indagar en la compleja relación entre las tecnologías digitales en los centros escolares y las políticas de descentralización, así como en las implicaciones del modelo pedagógico resultante. Los referentes teóricos se inscriben en el campo de la "nueva gestión pública" que pone el énfasis en la aplicación de indicadores, evaluaciones, regulaciones y aprendizajes autodirigidos como instrumentos del "management" de las organizaciones. ¿En qué medida estas políticas influyen en la reestructuración del modelo de enseñanza? Los datos y materiales de un proyecto de investigación, en cuyo trabajo de campo empleamos la encuesta, observación en centros, entrevistas, análisis documental y grupos de discusión, nos permiten fundamentar empíricamente la discusión sobre las tres cuestiones en las que desglosamos el objetivo principal. Concluimos que se descentralizan las decisiones sobre el 
equipamiento, se incrementan las tareas de gestión en detrimento de las didácticas y se recentraliza la gestión mediante la imposición de estándares.

Palabras clave: sistema de enseñanza; política de la educación; tecnología; transferencia de tecnología; descentralización; organización; organización de profesores.

\section{SUMMARY}

Our aim is to investigate the complex relationship between digital technologies in schools and the decentralization policies, as well as the implications of the resulting pedagogical model. The theoretical framework is grounded in the field of "new public management" which emphasizes the application of indicators, assessments, regulations and self-directed learning as the "management" instruments of organizations. To what extent these policies influence the restructuring of the teaching model? The materials and data of an research project, in which we employ fieldwork survey, observation in schools, interviews, documental analysis and discussion groups, allow us to empirically substantiate the discussion about the three issues which we break down the main objective in. We conclude that decisions on equipment are decentralized, management tasks are increased at the expense of teaching tasks and management is recentralized by the imposition of standards.

Key words: education system; educational policy; technology; technology transfer; decentralization; organisation; teacher's organisation.

\section{SOMMAIRE}

Notre objectif est d'étudier la relation complexe qui s'établit entre les technologies numériques aux écoles et les politiques de décentralisation, de même que les implications du modèle pédagogique qui en résulte. Le cadre théorique est inscrit dans le domaine qui correspond à la "nouvelle gestion publique ", laquelle met l'accent sur l'application d'indicateurs, d'évaluations, de réglages et d'apprentissages autodirigés, considérés comme instruments liés au " management " des organisations. Dans quelle mesure ces politiques influent sur la restructuration du modèle d'enseignement? Les données et les matériels d'un projet de recherche, travail de terrain dans lequel on a employé l'enquête, l'observation dans les établissements scolaires, les interviews, l'analyse de documents et les groupes de discussion, nous ont permis de fonder empiriquement la discussion sur les trois questions qui découpent l'objectif principal. On conclut donc que les décisions sur les équipements se décentralisent, que les tâches de gestion augmentent au détriment des didactiques et que la gestion se récentralise grâce à l'imposition de standards.

Mots clés: système d'enseignement; politique de l'éducation; technologie; transfert de technologie; décentralisation; organisation; organisation d'enseignants. 
ÁNGEL SAN MARTÍN ALONSO Y JOSÉ PEIRATS CHACÓN

Invisible el inspector reina como un espíritu; pero en caso de necesidad puede este espíritu dar inmediatamente la prueba de su presencia real.

(Betham, 1979, 37).

\section{INTRODUCCIÓN}

Resulta cada vez más evidente que la descentralización no es sólo la dispersión territorial del poder sobre la estructura y el sistema educativo, sino que conlleva la "entrega de poder y, consiguientemente, de responsabilidad" a las instituciones, centros docentes y demás agentes del sistema escolar (Embid Irujo, 2000, 25). En el caso del Estado español, la descentralización es introducida "por vía constitucional y parlamentaria, es decir, legal y política"; lo cual significa que se plantean como "principios no discutibles y legitimados por sí mismos, con independencia de su aplicación, prácticas y usos" (Viñao Frago, 1994, 55). La propuesta descentralizadora fue posible, según Puelles Benítez (2011), porque las fuerzas políticas y sociales de entonces fueron capaces de llegar a lo que califica como "consenso básico". Consenso que desde el principio afectaba más a los principios que a las prácticas y decisiones que se tomaban en los despachos, tanto de la administración central como de las autonómicas. De ahí que dicho consenso fuera desbordado de inmediato y hoy resulte apenas reconocible ni tampoco invocado ante los cambios que inducen las tecnologías en los centros.

Según el análisis de Viñao Frago (1994, 56 y ss.), "lo que ha sucedido en España ha sido la sustitución de un monocentrismo por un policentrismo en el que, por lo general, esos nuevos centros reproducen en su ámbito los esquemas y modos de funcionamiento del sistema precedente». En cualquier caso, se lleva al redactado de la Constitución de 1978 una propuesta de descentralización que Puelles Benítez $(1993,11)$ cataloga como "modelo intermedio". Un modelo en el que lo más significativo «es el reparto de competencias entre el gobierno central y las comunidades autónomas". El Estado se reserva las normas básicas sobre la ordenación general del sistema educativo o la fijación de una parte del currículo nacional, mientras que los gobiernos autónomos tienen competencias plenas sobre los centros docentes, el profesorado o la inspección del subsistema autonómico. En estas circunstancias es lógico que no exista un modelo único y universalmente válido de descentralización, sino que es algo cambiante. Entre otras razones porque "todo intento descentralizador genera antes o después vientos centralizadores y, viceversa..." (Viñao Frago, 1994, 34).

Las políticas en favor de la descentralización de los sistemas escolares, hoy en revisión, fueron apoyadas por los organismos internacionales, e incluso desde informes tan influyentes como el elaborado por la "Comisión Delors". De hecho, en este informe se recomendaba directamente la descentralización, porque así se 
fomentaría la participación de los agentes escolares y, sobre todo, se podrían aprovechar mucho mejor los recursos disponibles. Manifestación que, sin embargo, no es óbice para sostener: "la experiencia internacional demuestra que en los casos en que tiene éxito la descentralización, ésta se produce a partir de una administración central sólida" (Delors, 2001, 185). Tal vez se entienda mejor esta aparente paradoja si asumimos, como señala Lewkowicz $(2012,74)$, que "la globalización unifica el mundo desde el punto de vista del estímulo, pero las respuestas son diversificadas localmente». Hecho que constataremos en la relación entre equipamiento tecnológico y estrategias de control sobre las organizaciones que acogen los medios.

Ante el dilema que emerge la administración reacciona asumiendo lo que se conoce como la "nueva gestión pública", contando para ello con el inestimable concurso de las tecnologías de la información (Bush, 1986; Verger, 2012; Demazière, Lessard y Morrissetteet, 2013). Es muy revelador que en un informe programático de la UNESCO se concluya que "las TIC representan un paso decisivo en el proceso de democratización" porque permiten el acceso a los servicios públicos y a la información de las instituciones (Batista, 2003, 62). A partir de estos planteamientos surgen diversas cuestiones y una de ellas recorre todo este trabajo: ¿En qué medida la dotación de equipamiento tecnológico a los centros escolares impone una nueva relación entre la centralización y la descentralización?

\section{SOPORTE MACROPOlítico DE LAS TECNOLOGÍAS EN LOS CENTROS}

Una primera aproximación al trasfondo del interrogante anterior, nos la proporcionan los dictámenes de determinados organismos internacionales. Con frecuencia su ámbito de trabajo no es el educativo, sin embargo, sus propuestas legitiman determinadas actuaciones en los sistemas escolares, como la iniciativa ministerial Escuela 2.0. En este sentido resulta inevitable la referencia a la Meta 8.F de los Objetivos de Desarrollo del Milenio (ODM, 2014, 55 y ss.), se propone que, con la cooperación del sector privado en la transferencia de tecnología, se facilite el acceso a los beneficios de las nuevas tecnologías, especialmente potenciando la penetración en los países en desarrollo de la banda ancha, tanto fija como móvil, para sobrepasar la tasa actual del 6\% y 20\%, respectivamente. El Banco Mundial (Grupo del Banco Mundial, 2011), por su parte, en la Education Strategy 2020, bajo el subtítulo de "Aprendizaje para todos" propone que al igual que está sucediendo con las Tic en otros ámbitos productivos, deben aprovecharse las posibilidades que ofrecen aquéllas para accelerated learning and improved management of education systems.

En la última versión del documento Estrategia 2020, según Verger y Bonal (2011), el Banco Mundial no le confiere demasiada relevancia a las políticas de descentralización del currículum o a la formación del profesorado en Tic. Ahora focaliza toda la atención sobre la gobernanza, basada en la rendición de cuentas, en los incentivos por productividad y en el compartir responsabilidades entre los actores públicos y el sector privado (Innerarity, 2006, 209). Focalizar la mirada 
sobre estos tres aspectos supone introducir un modelo de gestión de los centros educativos basado en el conocimiento sobre su funcionamiento. Si bien, como destacan Verger y Bonal, en ningún momento se especifica en el documento del Banco Mundial qué relación hay entre el modo de gobernanza y el aprendizaje efectivo de los estudiantes. Y no la hay por la prevalencia de la impronta economicista y de mercado que inspira todo el desarrollo de la Estrategia 2020, tal como sostienen los autores citados.

Desde luego que esta propuesta sólo puede entenderse en pleno dominio de los programas neoliberales, especialmente en los servicios públicos. Perspectiva en la que se inscriben las siguientes palabras del influyente Drucker $(1993,68)$, cuando mantiene que ha de tomarse como oportunidad de innovación el hecho de que "la sociedad poscapitalista debe ser descentralizada; sus organizaciones deben ser capaces de tomar decisiones rápidas, basarse en la proximidad al funcionamiento, proximidad al mercado, proximidad a la tecnología, proximidad a los cambios en la sociedad [...]". Para este economista el proyecto de las organizaciones debe contribuir a cohesionarlas, hasta el punto que éstas puedan mantenerse incluso cuando sus valores no son del todo coincidentes con los de su entorno cultural.

En el informe coordinado por Delors $(2001,202)$ para la UNESCO, se afirma que "como instrumentos de educación de los niños y de los adolescentes, las nuevas tecnologías brindan una posibilidad sin precedentes de poder satisfacer con toda la calidad necesaria una demanda cada vez más amplia y cada vez más diversificada". Y poco más adelante la comisión responsable del referido informe recomienda que, habida cuenta de la revolución digital experimentada durante la última década, "la cuestión del empleo de las nuevas tecnologías en la educación es una decisión de carácter financiero, social y político y debe situarse en el centro de las preocupaciones de los gobiernos y de las organizaciones internacionales".

En efecto, mientras esos organismos se ocupan de articular políticas que traduzcan en prácticas tales "preocupaciones", desde el sector industrial toman posiciones y experimentan modelos de enseñanza calificados ya como electrónicos o digitales. Pero, ¿en qué consisten esos modelos de formación? A partir de las experiencias puestas en marcha con éxito por diferentes multinacionales (desde Total, Intel a IBM, pasando por DigitalThink), Gil (2001, 14) advierte que estamos ante "una auténtica reingeniería de la formación hecha ahora posible por la tecnología". Y bastantes páginas más adelante destaca que los componentes fundamentales de estos nuevos modelos serían: "Crear, gestionar, administrar los contenidos, difundir recursos de Web Based Training, seguir la trayectoria individual de los aprendices, gestionar los tutores online", todo lo cual conduciría a los Learning Management Systems (57). Como el propio autor señala, estos modelos de formación son posibles gracias a las casi ilimitadas prestaciones de tecnologías como la informática y las telecomunicaciones, que permiten tratar la información como nunca antes se había podido hacer. Plantean el acceso al conocimiento mediante códigos diferentes: "La revalorización del bricolaje en la cultura de la simulación incluye un nuevo 
énfasis en la visualización y en el desarrollo de la intuición a través de la manipulación de los objetos virtuales» (Turkle, 1997, 68).

Por otro lado, ante las llamadas de atención de organismos como Naciones Unidas o la UNESCO sobre el crecimiento imparable de la "brecha tecnológica" entre unos países y otros, desde la atalaya del MIT Media Lab, Nicholas Negroponte propuso en 2005 el proyecto de "One Laptop per Child" (OLPC) como medio para mitigar las diferencias. Proyecto que luego se ha difundido como el 1:1 o un ordenador por alumno, aunque ya no al precio de 100 dólares por ordenador como se aseguró al principio. Pero este visionario de las tecnologías no pensó únicamente en el proyecto OLPC como medio para facilitarles ordenadores a las niñas y niños más desfavorecidos, sino que concitó la complicidad de empresas del sector informático, de colegas como Seymour Papert quien le proporcionó el envoltorio pedagógico de orientación "constructivista" a su iniciativa y de colaboradores como algunos exitosos empresarios que mediaron en la negociación, para que varios gobiernos iberoamericanos y asiáticos firmaran contrato con la fundación que gestiona el OLPC.

En definitiva, estos organismos internacionales aportan los argumentos con los que legitimar determinadas políticas y prácticas. Ya sea al amparo de las políticas de aminorar las diferencias sociales, de mejorar la calidad de los sistemas escolares o de la innovación de las prácticas de enseñanza; el caso es que desde estas instancias se interfiere en las políticas nacionales de equipamiento tecnológico. Según todos los indicios, su apuesta es a favor de la descentralización en la toma de decisiones, como puso de manifiesto el programa Escuela 2.0 y la deriva posterior en el acceso a los contenidos digitales del currículo. Advertir la presencia de estos condicionantes nos parece fundamental para interpretar el sentido de los datos de campo, al tiempo que se desestima cualquier forma de determinismo.

\section{CAMBIOS EN LA ORGANIZACIÓN DEL TRABAJO DOCENTE Y DISCENTE}

La impronta neoliberal se hace patente en la innovación científica y tecnológica, especialmente en el campo de las tecnologías relacionadas con la información y la comunicación, para plasmar su ideario político en la organización del trabajo (Coriat, 1976; Braverman, 1978). La innovación en estos campos, sin duda, es uno de los factores explicativos del cambio estructural experimentado por la organización fordista de la producción, dando lugar a la aparición de las organizaciones flexibles. En este sentido Alonso $(2007,74)$ plantea que la "flexibilidad productiva" en la que nos hemos instalado significa que en la "cuantitativa y abaratadora producción fordista (más productos, más baratos y más trabajo), hemos pasado a una flexibilidad postfordista, en la que todos los medios fluctúan según ciclos muy rápidos del mercado". Fluctuaciones que afectan igualmente a la fuerza de trabajo, ya sea en su formación como en el acceso y permanencia en el puesto de trabajo. 
A este respecto se puede mantener que para la organización productiva, en la economía del conocimiento, los artefactos digitales representan algo semejante a lo que Coriat (1991) mantiene que supuso el cronómetro en el taylorismo. No sólo establecen y controlan el tiempo de ejecución de tareas, también «interiorizan" el conocimiento práctico del operario -es la tecnología incorporada- que se manifiesta mecanizando la gestión del proceso productivo. Lo cual no sucede por casualidad, sino como consecuencia de la división social del trabajo que fomentan determinadas políticas. Las mismas que, a pesar de la peculiaridad tecnológica de cada organización (Alcaide Castro, 1983; Beltrán Llavador, 2010), imponen la presencia de unos medios digitales con el propósito, no siempre explícito, de alterar la constitución de la organización receptora. El problema que aflora en el caso de la educación, con frecuencia ocultado, es que el "proceso productivo", por más atractivo que resulte, no es homologable al de producción de un cartel publicitario o del procesamiento de datos, como tampoco lo fue en la época de la escuela que aplicaba la "pedagogía por objetivos".

Con o sin connivencia política, nos encontramos que el modelo pedagógico emergente es el que se inspira, al decir de distintos analistas, en los tres principios más característicos de la producción flexible: justo-lo-bastante, justo-a-tiempo y coste-justo. Por tanto, confluyen en dicho modelo, de una parte, la ubicuidad de los nuevos medios tecnológicos y, por otra, el elevado potencial de procesamiento y cálculo de datos e informaciones. Lo cual le permite reactualizar el diseño de instrucción presentándolo a través de interfaces amigables para los aprendices. Se configuran en ese formato entornos en los que los docentes/discentes, ahora usuarios/clientes, interactúan para gestionar su participación en el proceso de enseñanza y aprendizaje, en ocasiones sin más apoyo institucional que el del logo de la plataforma. Recordemos la doble función del interfaz: expresa lo que se ha de hacer y a la vez registra lo que se hace, sin aplicar ningún reglamento democrático sino el inapelable código binario.

La proyección de los tres principios, señalados más arriba, sobre las organizaciones escolares implica adoptar un nuevo modelo para su gobierno. Es la "gobernanza" que reclama el Banco Mundial o el gerencialismo del que hablan otros autores. Para Drucker (1993, 51 y ss.), la "revolución de la gestión" producida en el último cuarto del siglo pasado ha conseguido relegar a un segundo plano los tradicionales "factores de producción", lugar que ahora ocupa el de "gestión". En la coyuntura actual este economista mantiene que la gestión es "proporcionar saber para averiguar en qué forma el saber existente puede aplicarse a producir resultados". Cualquiera que sea la misión de las organizaciones, éstas se constituyen en torno a la función de gestión por cuanto va a guiar y mejorar constantemente el proceso productivo. Modelo gerencialista que, sin embargo, según Gómez Llorente (2000) provoca la "erosión interna" de la organización y sus dimensiones instituyentes. En palabras de Walker (2002, 240), los modelos basados en el gerencialismo dan entrada a las élites económicas que se alejan considerablemente de los objetivos propuestos por las políticas que propugnan la descentralización en los sistemas educativos. 
A partir de las consideraciones precedentes sobre el modelo de gobierno de las organizaciones escolares, cabe plantear que la transferencia de tecnología, sobre todo la digital, tiene consecuencias que van más allá de las prácticas de aula. Por un lado, se observa una mayor descentralización en la toma de decisiones y, paralelamente a este proceso, otro en sentido inverso. Se advierte, por otro lado, una intensificación de las tareas de gestión que vendría exigida por la necesidad de realizar un control centralizado mediante estrategias telemáticas de aquello que se hace en los centros escolares. Extremo que aparece en boca de casi todos los entrevistados, pertenezcan o no a los equipos directivos, como comprobaremos más adelante.

\section{Planteamiento Metodológico}

Los materiales y datos de este trabajo proceden del proyecto Ticse $2.0^{1}$, cuyo propósito fundamental es identificar las opiniones, expectativas y valoraciones del profesorado de Educación Primaria y Secundaria hacia el Programa Escuela 2.0 y el uso de las Tic en su docencia, explorar qué tipo de prácticas de enseñanza se organizan en el contexto del aula y cómo se integran y coexisten con los materiales didácticos tradicionales, además de realizar un análisis comparativo de estos fenómenos entre las comunidades autónomas que participaron en el citado programa ministerial con otras no incorporadas al mismo. En definitiva, los once equipos de investigación trabajamos sobre estas cuestiones en nuestras respectivas comunidades autónomas, siguiendo las fases establecidas al inicio y revisadas periódicamente en las reuniones de coordinación. Los datos y materiales manejados aquí son los definitivos, y fueron elaborados en las dos primeras fases del citado proyecto:

Primera fase.- Explorar las opiniones, expectativas y valoración del profesorado con relación al Programa Escuela 2.0 o su equivalente autonómico. Tarea que realizamos a través de un cuestionario elaborado entre todos los equipos de investigación. El instrumento constaba de cinco dimensiones: TIC y práctica docente en el aula, TIC y centro escolar, TIC y profesorado, materiales utilizados en las aulas y valoración del programa de la comunidad autónoma. Estas cinco dimensiones se desarrollaron en 32 ítems cerrados y de opción múltiple, reformulados a partir del informe de tres jueces. La población del estudio la constituía el profesorado de primaria y secundaria de centros públicos; además debían ser usuarios de Internet para poder participar como sujetos en un estudio que aplica una e-encuesta

1. Trabajo vinculado al proyecto "Las políticas de un ordenador por niño en España. Visiones y prácticas del profesorado ante el programa Escuela 2.0. Un análisis comparado entre comunidades autónomas". Secretaría de Estado de Investigación. Dirección General de Investigación y Gestión del Plan Nacional de I+D+i. Subdirección General de Proyectos de Investigación del Ministerio de Ciencia e Innovación (REF. EDU2010-17037). 
(Cubiles de la Vega, Muñoz Conde, Muñoz Pichardo y Pascual Acosta, 2002). El cuestionario se alojó dos meses (febrero y marzo de 2011) en la plataforma del Instituto de Tecnología Educativa (ahora INTEF) a fin de facilitar el acceso por la red desde cualquier centro escolar del Estado que participara en el programa ministerial.

Los cuestionarios recogidos y que resultaron válidos configuraron una muestra definitiva de 5.161 sujetos de 15 comunidades autónomas. De los cuales el 53,2\% eran mujeres y el 48,8\% varones; el 60,3\% trabaja en la etapa de primaria, el 37,9\% en secundaria y el resto en ciclos formativos. Con las respuestas dadas a los cuestionarios se ha llevado a cabo el análisis de las tablas de distribución de frecuencias y los estadísticos descriptivos de cada una de las variables del cuestionario, el ANOVA de un Factor, la prueba T para muestras independientes, tablas de contingencia y coeficiente chi cuadrado ( $\chi 2)$. Dichos análisis fueron realizados a través del programa SPSS.18 y SPSS. 19 win (Area, 2012).

Segunda fase.- Identificar y analizar el tipo de actividades didácticas que se organizan en el aula con los nuevos medios, prestando atención a su impacto en la metodología de enseñanza y cómo se integran y coexisten con los materiales didácticos tradicionales. A tal propósito cada equipo realizó en su respectiva comunidad autónoma varios estudios de casos en los que se aplicaban observaciones, entrevistas, grupos de discusión, análisis de los documentos facilitados por los centros o conseguidos en la Red. Todas estas herramientas metodológicas están vinculadas a un marco de transformación paradigmática en el que la etnografía viene a requerir un redimensionamiento de sus conceptos y la adaptación de sus principios básicos al estudio de las relaciones sociales mediadas por las tecnologías digitales, fundamentalmente Internet. En definitiva, es el planteamiento que algunos metodólogos adjetivan como etnografía virtual (Mosquera, 2008, 538 y ss.). Los materiales grabados y luego transcritos se analizaron mediante el Atlas-Ti-7 para identificar las categorías con mayor frecuencia y en torno a ellas hemos reconstruido las unidades de sentido más pertinentes para nuestros propósitos.

Los datos y materiales recabados bajo las condiciones descritas, constituyen la base con la que nos proponemos responder en el quinto apartado a las tres cuestiones planteadas a continuación. Concretamente los datos corresponden a la dimensión "TIC y centro escolar" del cuestionario, mientras que la voz del profesorado se extrae de las transcripciones de las entrevistas y grupos focales realizados. De modo que a partir del planteamiento teórico precedente y de los datos recabados, planteamos las tres preguntas que guiarán la indagación y discusión a propósito de las implicaciones que tienen las tecnologías sobre la organización y gestión de los centros escolares:

a) ¿La implementación del Programa Escuela 2.0 generó iniciativas autonómicas convergentes o tendieron a la diferenciación?

b) ¿En qué medida las tareas diarias del profesorado están más vinculadas a la gestión de la información que específicamente con la didáctica?

c) ¿El modelo de gobernanza se centra en la gestión de estándares y homologación de resultados como estrategia de recentralización? 


\section{EXPOSICIÓN Y COMENTARIO DE RESULTADOS}

El Gobierno incorpora al Programa Nacional de Reformas la iniciativa Escuela 2.0 con el propósito de facilitar un ordenador a cada alumno. Pero son las comunidades autónomas quienes tienen las competencias para decidir sobre las condiciones particulares de implementación. Y deciden hacer cosas diferentes. Por otra parte, como el cronómetro en el taller, la informática en las escuelas impone una nueva forma de organizar el trabajo y las relaciones sociales en torno al quehacer y saber pedagógico. Parafraseando al ya citado Coriat (2001, 35), valdría decir que si la máquina informática se apropia de las habilidades asociadas al oficio docente, entonces la actividad de enseñar se puede reproducir a gran escala y sin necesidad de ningún saber previo. Lo cual respondería, según ha denunciado Cuban (1986), al viejo sueño de encontrar un artefacto más eficiente y más barato para transmitir información que el profesorado.

En la actual reedición del citado sueño, el problema se centraría en articular y gestionar los "servicios" que desde el exterior prestan otras agencias ajenas al proyecto pedagógico de los centros. Tal sería el caso de actividades como la visita virtual a un museo desde la materia de historia del arte o de ciencias naturales, el concurso educativo de una entidad financiera, el acceso a una dirección web para consultar /descargar material relativo a los contenidos curriculares o la creación de un canal en YouTube para alojar los vídeos de graduación. ¿De qué legitimidad epistémica gozarían estas ofertas "externalizadas" y cómo se gestionarían en las aulas?

No cabe duda de que la presencia de las tecnologías digitales en los sistemas escolares tiene innumerables ventajas, pero también plantea no pocas incógnitas. Dicho esto, en lo que sigue trataremos de responder a cada uno de los tres interrogantes planteados más arriba, aunque las respuestas no pueden ser en términos dicotómicos. Más bien trataremos de construir un relato en el que se articulan los datos procedentes de los ítems del cuestionario (dimensión de centro y en menor medida de la de profesorado) con los obtenidos a través de las entrevistas y observaciones.

\subsection{Acciones semejantes en territorios distintos}

Respecto al primer interrogante planteado más arriba, traemos a colación el titular con el que un diario nacional de información económica, encabezaba un artículo sobre el Programa Escuela 2.0: "El portátil del cole divide España" ${ }^{2}$ A nuestro entender, el titular pone de manifiesto el alcance político del citado plan, tanto por lo que se refiere a cómo se diseñó y negoció como a la receptividad que las iniciativas de la administración central tiene entre las autoridades autonómicas, depositarias de las competencias en materia de educación. Lo cual supone asumir

2. Consultado el 30 de mayo de 2014, http://goo.gl/ywk6zG. 
que hay 17 formas posibles de interpretar y aplicar el mencionado programa, por lo menos de 15 de ellas hemos obtenido en la investigación evidencias empíricas sobre sus actuaciones de convergencia o divergencia con la propuesta ministerial.

Es un hecho que cada comunidad autónoma interpretó y aplicó a su modo el programa impulsado por el Gobierno central, exhibiendo con ello el hecho diferencial aunque todas siguieron un patrón parecido: dotar de tecnología informática a los centros escolares. En esa dirección, nada menos que el 94,3\% de los encuestados consideran que estos programas tienen algún efecto en la marcha y mejora de los centros (Ítem 29). Ahora bien, para el 75,5\% dicho efecto se focaliza casi exclusivamente en el aumento de recursos tecnológicos en los centros.

Otro hecho a destacar es que si había diferencias en el punto de partida, tras la "aportación autonómica" al programa ministerial, tales diferencias persisten e incluso en algún caso aumentan. Ante los datos facilitados por el propio ministerio (MECD, 2013, 29), observamos en la siguiente gráfica que, por ejemplo, la Comunidad de Madrid ofrece un ordenador para cada 6,2 estudiantes en el curso 2011-2012, mientras que en el otro extremo está Extremadura, con 1,5 alumnos por ordenador. Parecidas diferencias se mantienen también en lo relativo a la calidad de la conectividad de los centros, especialmente los de titularidad pública. Todo lo cual responde a que la descentralización de las competencias educativas no ha conseguido reequilibrar las diferencias estructurales pese al esfuerzo de convergencia realizado durante las últimas décadas (Pérez Esparrells y Morales Sequera, 2012).

Figura 1. ORDENADORES EN CENTROS PÚBliCOS (MECD, 2013)

(1) Se consideran los ordenadores destinados preferentemente al profesorado y a la docencia con alumnos.

\section{Número medio de alumnos por ordenador ${ }^{(1)}$ en centros públicos, por comunidad autónoma. Curso 2011-2012}

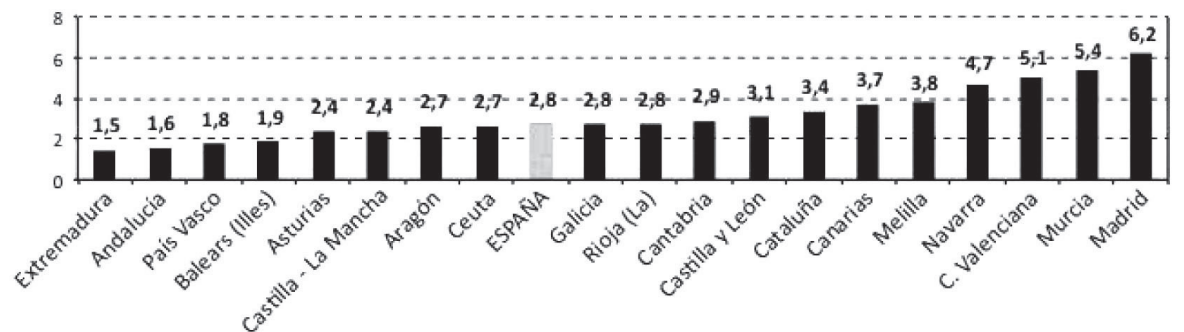

(1) Se consideran los ordenadores destinados preferentemente al profesorado y a la docencia con alumnos.

De modo que las reacciones a la iniciativa Escuela 2.0, como cabía esperar, fueron muy diversas y motivadas por razones diferentes. El signo político de 
los respectivos gobiernos autonómicos, sin duda, fue uno de los desencadenantes, pero también el PIB de cada una de ellas, su deuda y estado de ejecución presupuestaria del ejercicio en curso, el 2009. Téngase en cuenta que el programa se presenta en mayo de ese año y comenzó a ejecutarse cuatro meses más tarde, en el curso 2009-2010, teniendo que aportar cada comunidad autónoma el 50\% de la inversión prevista, la otra parte el Gobierno central. No olvidemos que cada comunidad tenía en marcha su propio plan o programa de TIC para sus centros escolares, a lo que ahora debían añadir el que se proponía liderar el Ministerio de Educación. Tal vez por esto, la directora general de Innovación Tecnológica de la Conselleria de Educación de la Generalitat Valenciana nos hizo la siguiente valoración en una entrevista: "El Escuela 2.0 es un modelo al que no se le puede mover ni una coma. Nos lo ofrecen cerrado, como si no tuviéramos nada que aportar".

Por unas u otras motivaciones, cada comunidad autónoma interpretó y adaptó el programa ministerial conforme a sus prioridades políticas y/o educativas, dando lugar así a ofertas diferencias. Pese al intento de adaptación, casi un 20\% del profesorado encuestado manifestó estar muy en desacuerdo con las iniciativas políticas adoptadas, frente a un 10\% que reconocía estar totalmente de acuerdo (ítem 27). De modo sintético, el espectro resultante de iniciativas diferenciadas por las administraciones autonómicas lo podemos incluir en uno de estos tres grupos:

a) Hubo comunidades que rechazaron desde el primer momento incorporarse a dicho plan, tal fue la decisión de Madrid, Valencia y Murcia ${ }^{3}$, impulsando en contrapartida planes propios pero sin el apoyo económico del Ministerio. La Generalitat Valenciana puso en marcha la iniciativa Centros Educativos Inteligentes (CEI), cuyo objetivo era atender tecnológicamente a todos los ámbitos del centro. El proyecto se experimentó en dos centros, luego se amplió a 16 más y cuando se pretendía abrir la convocatoria para más de medio centenar de centros, el proyecto desapareció sin ningún tipo de evaluación ni explicación pública.

b) Un segundo grupo estaría constituido por aquellas comunidades que desde el principio aceptaron entrar en el plan, integrándolo en iniciativas propias. Tal sería el caso de Cataluña, Andalucía, País Vasco o Asturias, por lo general son comunidades muy activas en cuanto a la aplicación de las tecnologías en sus centros escolares. Bajo estas coordenadas se activaron iniciativas como Escuela2.0-Asturias, Escuela TIC 2.0 en Andalucía o EduCAT1x1 en Cataluña.

c) Otro grupo de comunidades, aunque reticentes, aceptaron incorporarse más lentamente y aspiraban a disponer de "aulas digitales». En semejante

3. La Comunidad de Murcia no se sumó inicialmente a Escuela 2.0, sin embargo, rectificó y se incorporó a partir del curso 2010-2011. Consultado el 23 de abril de 2014 http://goo.gl/CVwScN. 
tesitura se situaron comunidades como Galicia, Cantabria, Extremadura o Canarias, que se sumaron en el curso 2010-2011 al programa ministerial con todas las consecuencias, insertándolo en sus planes de actuación con nombres tan poco reconocibles como el de Abalar en Galicia, Estrategia Red XXI en Castilla y León o Mochila Digital en Castilla-La Mancha.

A modo de consideración final destacaríamos, a partir de lo expuesto en los párrafos precedentes, que el patrón seguido se caracteriza por la siguiente secuencia: los organismos internacionales establecen las metas a seguir respecto a cada generación tecnológica, los gobiernos nacionales las traducen en proyectos de actuación (Escuela 2.0 y ahora Agenda Digital), la comunidad autónoma diseña la implementación y los centros, último escalón de la cadena, lo ponen en marcha con el entendimiento y los medios que tienen. ¿Hasta qué punto, tal como observa Stiglitz (2003, 359), estas estrategias socavan las estructuras del poder político del Estado en favor de instancias económicas globales?

\subsection{Reestructuración de roles y tareas de los agentes escolares}

Por lo que se refiere al segundo interrogante, destacamos que uno de los ámbitos más sensibles tanto a la presencia de las diferentes tecnologías como a la descentralización es el relativo a las relaciones laborales que constituyen la organización de los centros escolares. Pese a que casi nadie cuestiona la relevancia de la función docente, cada vez aparece menos nítido su perfil laboral y reconocimiento profesional. Algo parecido podría decirse también en relación a la función discente, pese a su proclamado empoderamiento. La proliferación de recursos en las aulas y las experiencias dispares con estos medios de los agentes escolares generan un contexto de enseñanza y aprendizaje en el que el profesorado pierde credibilidad y sobre todo autoridad. Uno de los profesores entrevistados, docente en un ciclo formativo (IES-2), señalaba en relación a esta categoría de análisis que "ya no podemos ser predicadores del conocimiento, porque el ordenador lo recuerda mejor que nosotros y lo expone de forma más bonita" (P1CF). Por otra parte, a la función docente se le van adhiriendo tantas nuevas tareas como aplicaciones tecnológicas se incorporan al quehacer cotidiano en los centros escolares.

Ahora bien, los datos empíricos ponen de manifiesto que no sólo cambian los roles y tareas de los agentes escolares, cambia también la naturaleza misma del trabajo que ya no es sólo el que se realiza en la fábrica, sino en los hogares, las calles o las escuelas. El capitalismo más avanzado ha conseguido que la producción informacional es "trabajo", ya sea para quien realiza un determinado producto como para quien lo consume (Míguez, 2008, 16). En este caso, tanto el docente como el aprendiz realizarían un trabajo complejo, que no será físico ni tan rutinario como el de la cadena. Frente a una pantalla interactuarán con la interfaz que le requiere interpretar símbolos y ejecutar instrucciones, por lo que es un trabajo que se podría calificar como eminentemente cognitivo. La figura siguiente 
refleja bastante bien la tipología de tareas que el profesorado reconoce realizar con mayor frecuencia y que resumimos como "gestión de información".

\section{Figura 2. ACCiOnes DeSARROlLadas CON TiC EN la Clase (ÍTEM 12)}

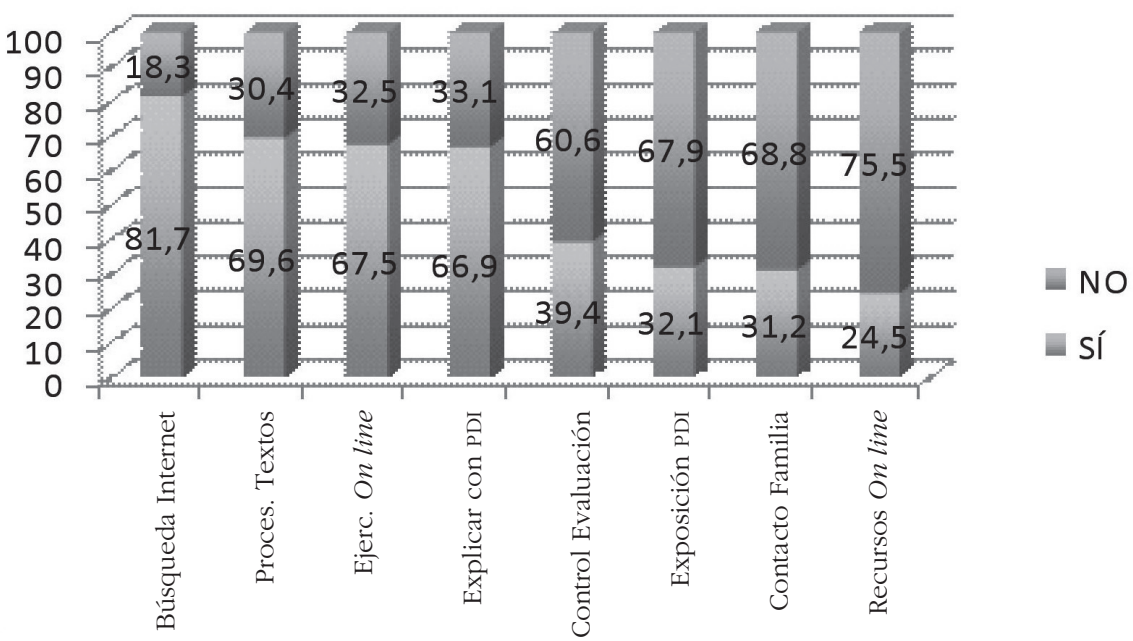

Para Tenti Fanfani $(2010,44)$ tanto la nueva coyuntura política como el desarrollo de las TIC están generando la aparición de nuevos trabajadores y profesionales de la educación. Aunque considera muy difícil anticipar el perfil del docente en las escuelas de dentro de unos pocos años, sí anticipa que dicho "oficio" lo ejercerán distintos profesionales que compartirán la responsabilidad con el profesorado. Los nuevos maestros habrán de asumir tareas como el diseño de programas virtuales de formación, producir contenidos, activar y gestionar redes virtuales, interaccionar con otras personas en contextos muy diversos o realizar la evaluación valiéndose de sofisticadas herramientas tecnológicas. Para otro observador de este proceso de cambio, el profesorado deja de ser "un mero transmisor de conocimientos para desempeñar el rol de facilitador, o mediador de procesos de aprendizaje..." (Díaz Becerro, 2009, 3). Con otras palabras recreaba este perfil laboral uno de los profesores participantes en el trabajo de campo:

Hay un importante trabajo previo, antes de la clase has de preparar el repertorio de materiales, cortando de aquí y de allá, revisando los ejercicios. Es un trabajo estimulante pero más intenso que antes, puesto que el libro ya te lo daba todo hecho... Luego en la clase no hay por qué estar uno hablando continuamente, yo dedico mucho tiempo a la tarea de observar a los alumnos, a hablar con ellos para saber si han entendido o no los ejercicios que hacen. [...]. También tenemos 
que hacer de animadores, apoyar al grupo y tratar que todos se impliquen en la actividad, que la hagan y a ser posible cada vez en menos tiempo. Y esto exige hablar con ellos, animarlos y todas esas cosas que muchas veces desconocemos. La capacidad de decidir estos momentos es mía, no de la máquina (PM del IES-2).

Y si cambia el rol del docente también debe hacerlo en parecido sentido el del estudiante, para que encaje en el nuevo protocolo de organización del trabajo (el perfil que el profesorado le atribuye es muy parecido al suyo, tal como se desprende de las entrevistas realizadas). En coherencia con el modelo, se transfiere un especial protagonismo al usuario, por cuanto su actividad es lo que confiere sentido al conjunto del entramado. Argumentos en favor de este vuelco hay muchos pero tomamos uno como muestra: «[...] es el propio alumno el que va construyendo su conocimiento a través de las interacciones con otros estudiantes como él, con el profesor y con el material didáctico disponible", principalmente en la plataforma virtual (Díaz Becerro, 2009, 4). Observemos en este sentido lo que mantiene un profesor de primaria (CEIP-3):

Yo no me he iniciado en el tema, yo sé que, por ejemplo, en el área de inglés los niños cuelgan actividades en la plataforma Moodle. Yo todavía no me manejo bien en ese tema pero sí que es verdad lo que comentan ellos, que el material y con los trabajos que preparan en PowerPoint y todo eso, aprenden más o al menos trabajan más... (PP1).

Por otra parte y hasta no hace mucho, el sistema escolar regulaba, a partir de los manuales, tanto los contenidos a transmitir como las prácticas de aula, sin embargo, en estos momentos las fuentes de acceso son prácticamente ilimitadas. Circunstancia que plantea importantes y novedosos problemas: tanto de orden pedagógico, porque no es lo mismo trabajar con los libros de texto que ante una pantalla interactiva con los contenidos digitalizados, de orden tecnológico relativo al soporte, al formato y al medio, así como de gestión de lo que se enseña y certifica en el sistema escolar, a partir de unidades didácticas accesibles en repositorios, objetos de aprendizaje, el libroweb o el e-book. En un grupo de discusión con docentes de primaria (CEIP-3), se argumentaba:

[...] yo creo que cada vez más el libro de texto tradicional se nos está quedando cojo [...] A nivel de recursos digitales y de información, de recursos que se puedan ir creando, yo creo que cada vez los profesores deberíamos ser más autónomos a la hora de crear nuestros propios recursos. Podemos apoyarnos en los que ya existen en la red, lógicamente, aprovechamos estos recursos y los compartimos con los demás colegas (PP2).

En definitiva, la Figura 2 dibuja con bastante precisión el perfil didáctico del docente y que sintetizamos con la expresión "gestor de información", lo que suscita no pocas cuestiones de orden teórico respecto a la naturaleza de la enseñanza y función de la escuela. Por otra parte, los contenidos y el acceso a ellos plantea nuevos problemas. Entre otros, el que ahora las editoriales han de competir con 
iniciativas particulares del profesorado, administraciones y agentes sociales que ofertan sus propios diseños de contenidos (Varela Mallou, 2008). En este contexto desregulado, ¿cómo se validan científica y epistemológicamente los contenidos en formato digital de libre acceso?

\subsection{La gestión para la obtención de resultados estandarizados}

Las organizaciones clásicas, según Castells (2009, 47 y ss.), han de entenderse como una burocracia jerárquica basada en "la integración vertical de los recursos y de los sujetos, como expresión del poder organizado de una élite social». Sin embargo, en esta disposición organizativa se incorporan unas tecnologías que operan principalmente con la lógica de redes, bien porque a través de aquéllas se crean comunidades virtuales o bien porque sirviéndose de ellas la comunidad escolar real pasa a formar parte de una red de trabajo. En ambos casos las redes operan, siguiendo a Castells, conforme a un protocolo binario de "inclusión-exclusión" que genera complejas estructuras de comunicación establecidas en torno a "un conjunto de objetivos que garantizan al mismo tiempo, unidad de propósitos y flexibilidad en su ejecución gracias a su capacidad para adaptarse al entorno operativo". Entorno que permite instaurar sistemas de control a los que sólo tendrán acceso las instancias que promovieron la descentralización de la organización. De ahí que se recomiende en un informe de la Unión Europea (EU, 2013, 156), en relación con las TIC, implementar desde las instancias centrales políticas de "gestión eficiente del aprendizaje".

Un centro público de la Comunidad Valenciana, tomado a modo de ejemplo, puede tener simultáneamente y de hecho muchos lo tienen, acceso a una plataforma para la gestión administrativa (ITACA), otra para la académica (Mestre a casa), ambas facilitadas por la Generalitat. Los centros además contratan los servicios de Tecnausa-SGD para la gestión cotidiana de faltas, la instalación de plataformas como Moodle (por citar la más extendida) para la actividad académica, y además el blog autónomo del departamento de orientación. Semejante proliferación de instancias y recursos tecnológicos genera no pocos conflictos, pues no siempre cuentan con la legitimidad y/o pertinencia para realizar las actuaciones más adecuadas a los propósitos de la institución. En este sentido cabe plantear por qué a los estudiantes se les prohíbe el móvil en clase, pero al profesorado no, o si el "consentimiento informado" es suficiente para que una empresa privada tenga ficheros con los datos académicos y disciplinares de los estudiantes o los registros de las videocámaras instaladas en la entrada del centro. Esa diversidad de prácticas se resume en la siguiente tabla de datos. 
ÁNGEL SAN MARTÍN ALONSO Y JOSÉ PEIRATS CHACÓN

IMPACTO DE LAS TECNOLOGÍAS DIGITALES EN LA DESCENTRALIZACIÓN DEL SISTEMA ESCOLAR

TABla 1. USO DOCENTE DE LOS SERVICIOS WWw (ÍTEM 24 EN \%)

\begin{tabular}{|l|c|c|c|c|c|c|c|}
\hline & $\begin{array}{c}\text { NAVEGA } \\
\text { WEB }\end{array}$ & $\begin{array}{c}\text { CORREO } \\
\text { ELECTRÓNICO }\end{array}$ & $\begin{array}{c}\text { FORO/ } \\
\text { CHAT }\end{array}$ & BLOG & $\begin{array}{c}\text { REDES } \\
\text { SOCIALES }\end{array}$ & $\begin{array}{c}\text { AULA } \\
\text { VIRTUAL }\end{array}$ & $\begin{array}{c}\text { PORTALES } \\
\text { EDUCATIVOS }\end{array}$ \\
\hline $\begin{array}{l}\text { Todos } \\
\text { los días }\end{array}$ & 66,6 & 69,3 & 6,9 & 11,1 & 13,1 & 8,5 & 19,7 \\
\hline $\begin{array}{l}\text { Varios días/ } \\
\text { semana }\end{array}$ & 17 & 15,3 & 7,2 & 12,5 & 8,2 & 11,9 & 29,7 \\
\hline $\begin{array}{l}\text { Varios días/ } \\
\text { mes }\end{array}$ & 6,9 & 7,3 & 16,8 & 23 & 11,8 & 21,9 & 27,3 \\
\hline $\begin{array}{l}\text { Alguna ver/ } \\
\text { curso }\end{array}$ & 2,6 & 2,5 & 22,7 & 22,5 & 16 & 19,1 & 11,9 \\
\hline Nunca & 2,1 & 0,9 & 41,1 & 25,1 & 32 & 5,5 & 11,4 \\
\hline
\end{tabular}

Pese a disponer de un buen equipamiento tecnológico, tal como ponen de manifiesto los primeros ítems de nuestro cuestionario (ítem 10 y 11), luego la intensidad de uso no se corresponde con el material disponible. Al observar la tabla precedente advertimos que prevalece el uso de las web y el correo electrónico, herramientas que según el ítem 16 no se utilizan demasiado en la comunicación con otros centros ni tampoco con las familias del centro. En cambio, una gran mayoría del profesorado encuestado considera que estos medios motivarán a los alumnos, razón por la que no tendrán dificultad en controlar la marcha de los estudiantes en clase (80,7\% en el ítem 29), aunque algo más de la mitad del profesorado reconoce que estas herramientas le facilitará la evaluación de los aprendizajes (ítem 12). Asimismo destacamos que están bastante interesados por la comunicación con la administración, al menos casi un tercio de los encuestados responde en este sentido. Sobre el particular la secretaria de un instituto nos decía: "Antes pensaba que con estas máquinas se acabaría la burocracia del papel. Ahora vivo en propia carne cómo crece cada día... La mayor parte de mi tiempo lo dedico a hacer este tipo de gestiones burocráticas con el ordenador" (S1).

Aunque de momento conviven en las instituciones escolares la lógica jerárquica con la reticular, no sin provocar importantes controversias, se evoluciona hacia un modelo de gestión -management-, en el sentido tecnocrático del término, mediante el cual garantizar que los distintos nódulos cumplen su parte. No parece que, en este contexto, se consolide lo que algunos califican como "modelo de gestión no jerárquica", tal como señalan Díaz, Civís y Longás (2013, 219), más bien está sucediendo algo distinto. La organización "desconcentrada" del trabajo requiere núcleos estratégicamente posicionados para controlar el proceso, tal sería el caso, por ejemplo, de las evaluaciones PISA a partir de indicadores previos o las evaluaciones diagnósticas establecidas por la reciente ley de educación (LOMCE). Como también lo es la siguiente situación expuesta por el coordinador TIC de un colegio (CEIP-4) respecto a las "competencias" del servicio técnico de la Conselleria: "Ellos nos solucionan muchos de los problemas por el hecho de que se conectan de forma remota a nuestro servidor, a lo mejor es un proceso que no 
has adaptado bien o ha dejado de funcionar [...] y ellos lo solucionan. Pero también hacen cosas, como desconectar el servidor, sin que nos avisen que lo van a hacer, que me parecen intromisión... Sobre todo porque nunca te dan explicaciones". De modo que el modelo de enseñanza así concebido no escapa a un tipo de gestión más centralizado, esto es: "El movimiento centrífugo de la producción se equilibra mediante la tendencia centrípeta del mando" (Hardt y Negri, 2002, 276).

La instancia con autoridad y capacidad de control ya no depende tanto del liderazgo socialmente construido como de unas habilidades de carácter técnico y especializado en algunos fragmentos de la Red. De este modo aparece el "técnico" (asesor, coordinador de informática o el autodidacta más dispuesto), de quien depende el funcionamiento del equipamiento tecnológico (en torno al 90\% de los encuestados defiende la presencia de la figura de la coordinación Tic en los centros [ítem 18]. Consideran "muy necesaria" o "bastante necesaria" su presencia para "impulsar y mejorar el uso de las tecnologías en los centros"), pero también al especialista en dificultades de aprendizaje, al asesor en formación o al evaluador aplicando pruebas diagnósticas. Es llamativo el hecho de que cada vez sean más las gestiones ordinarias (contables, administrativas, certificaciones, disciplinares y académicas) que se realizan exclusivamente mediante entornos tecnológicos.

Una de las respuestas para coordinar organizadamente todas estas variables, es lo que se viene llamando gobernanza o reforma gerencial (Verger, 2012, 5 y 8). Para este autor los principios invocados por estas reformas son los de elección escolar, la autonomía de gestión de los centros, la competencia y gestión por resultados, etc. Con ello lo que se persigue es colocar al sector privado en el sistema público de educación. El argumento es que, según Verger, "el sector público debería aprender de la cultura gerencial del sector empresarial y adoptar sus normas, valores y técnicas". Una de sus estrategias más profusamente empleadas son las evaluaciones externas "cada vez más intrusivas y punitivas". A partir de los resultados de estas evaluaciones, en principio, para adoptar decisiones sobre la organización o sobre cualquiera de las intervenciones realizadas. Como ya señalara Gimeno Sacristán (1994, 12), las evaluaciones externas lograrían recentralizar el currículum desregulado, extremo que las tecnologías hacen más factible por la cantidad de datos que acumulan. Datos que se convierten en la materia prima de un nuevo modo de gobierno de los centros escolares, extremo apuntado en la tercera pregunta de investigación de este trabajo.

\section{Conclusiones}

La descentralización del sistema educativo apoyada en las iniciativas de transferencia de tecnologías genera una compleja bipolaridad multicentros y pocas cuestiones de orden teórico. Nos encontramos, por un lado, con que las administraciones públicas ceden ámbitos competenciales a distintos agentes que los ejercen para contestar a la autoridad central. Sin embargo, hemos visto que tanto las grandes líneas de actuación como los estándares sobre procedimientos 
y resultados a conseguir por las organizaciones escolares les vienen impuestos desde instancias macropolíticas. Los datos recabados sobre la iniciativa 1 a 1 de la administración central confirman que las autoridades autonómicas perfilan planes de actuación muy parecidos entre sí. Salvo el nombre todos ellos coinciden, según valora el profesorado y se constata en las observaciones, en mejorar el equipamiento tecnológico de los centros. Equipamiento que les sirve para multiplicar tareas de gestión de la información de docentes y discentes, pero no mejoran la comunicación ni el rendimiento académico de los estudiantes.

Los datos y documentos que acompañan al Programa Escuela 2.0 -adviértase que adjetivan la iniciativa política con el nombre de una generación de tecnologíainvitan a repensar lo que está cambiando en el interior de los centros, tanto en las formas de gestionar su "autonomía" como en las formas de organizar el trabajo en las aulas. Invitan también a repensar la legitimidad de los poderes que ocupan el "no lugar" de desarrollo de la enseñanza y del aprendizaje, así como identificar el "capital intelectual" que se está reproduciendo y distribuyendo con el apoyo de las tecnologías. Circunstancia que exige reclamar para la institución escolar no los viejos esquemas de gobierno, sino otros mucho más imaginativos que integren los medios tecnológicos hoy disponibles, con los preceptos de una democracia más compleja que no renuncia a los principios de equidad y rigor del conocimiento. Además de activar instancias de intermediación y procedimientos de participación mediante los cuales salvaguardar el compromiso moderno de la institución escolar con el saber y la cultura.

Emerge así un campo pedagógico en el que la autonomía de centro quedaría supeditada a fuerzas ajenas a sus instancias organizativas y a los controles democráticos. De modo que la descentralización, con el apoyo de las tecnologías transferidas, convierte a los centros escolares en espacios que se gestionan no tanto por los principios de la política de interés público sino por los que emanan del mercado. Todo ello sin que la educación pierda su carácter de derecho público fundamental.

Es cierto que últimamente los organismos internacionales ya no hablan de la descentralización del currículum, como apuntamos más arriba, ahora el énfasis se focaliza sobre la "gestión del conocimiento" que suplanta al método de enseñanza, a la didáctica, tal como la hemos venido entendiendo hasta ahora. Entre otras razones, porque el aprendiz se ha empoderado hasta el punto de convertirse en el "enseñante", al ser el "nativo digital". La desinstitucionalización inherente a este planteamiento es lo que suscita el siguiente comentario de un director: "Internet nos plantea problemas tan complejos y tan nuevos que ahora necesitamos una asesoría jurídica para adoptar determinadas decisiones o permitir algunas cosas en la red" (IES-2). En definitiva, la descentralización en la toma de decisiones hace emerger modelos de gestión basados en resultados mediante unas tecnologías que operan con protocolos tecnocráticos y altamente centralizados. ¿Tal coyuntura podría interpretarse como un intento encubierto de recentralización? Sin duda, y por ello hemos de seguir trabajando en esa línea pues se introducen cambios 
significativos en el acceso a un derecho fundamental de ciudadanía como es el de la educación.

Vivimos momentos de transición en los que resulta razonable asumir la convivencia entre la organización fordista de la escuela con las plataformas virtuales. Lo cual, a nuestro entender, plantea al menos un doble desafío a afrontar durante este tiempo de incertidumbre. Por un lado, continuar analizando con rigor y sin servidumbres tecnicistas la micropolítica de los centros escolares, abocados a resolver las contradicciones que en el quehacer diario les plantean los dispositivos de producción y reproducción del conocimiento. Especialmente en estos momentos en que se fomenta en los centros el acceso digital a los contenidos del currículum. Por otra parte y dado que esta coyuntura viene impuesta por el modelo político hegemónico en la actualidad, no queda otra salida que reivindicar la prevalencia de la institución escolar como instancia de intermediación social y garante de una forma de escolaridad a mantener, simplemente porque se muestra más solidaria que las ofrecidas en soporte digital. Si bien habrá que revisar y reformular el modelo teórico en el que sustentar las prácticas de aula en una institución desregulada pero con fuertes controles sobre los resultados.

\section{REFERENCIAS BIBLIOGRÁFICAS}

Alcaide Castro, M. (1983) Las nuevas formas de organización del trabajo. Madrid, Akal.

Alonso, L. E. (2007a) La crisis de la ciudadania laboral. Barcelona, Anthropos.

Alonso, L. E. (2007b) Las nuevas culturas del consumo y la sociedad fragmentada. Pensar la Publicidad, 1 (2), 13-32.

AREA Moreira, M. (dir.) (2012) La visión y opinión del profesorado sobre el Programa Escuela 2.0 en España. Un análisis por comunidades autónomas. Informe final del primer estudio. Consultado el 10 de julio de 2014. http://goo.gl/jmC3X5

BATISTA, C. (2003) Las TIC para la gobernabilidad. Brasilia, UNESCO.

Beltrán LlaVADOR, F. (2010) Organización de instituciones socioeducativas: imposibilidad $y$ desórdenes. Valencia, Reproexpres Ediciones.

Bentham, J. (1979) El Panóptico. Madrid, La Piqueta.

Braverman, H. (1978) Trabajo y capital monopolista. México, Nuestro Tiempo.

Bush, T. (1986) Theories of Educational Management. London, Harper \& Row.

Coriat, B. (1976) Ciencia, técnica y capital. Madrid, Blume Ediciones.

CORIAT, B. (1991) El taller y el cronómetro. Madrid, Siglo XXI.

Castells, M. (2009) Comunicación y poder. Madrid, Alianza Editorial.

Cuban, L. (1986) Teachers and Machines. The Classroom Use of Technology. New York, Teachers College Press.

Cubiles de la Vega, M. ${ }^{a}$ D.; Muñoz Conde, M. M.; Muñoz Pichardo, J. M. y Pascual Acosta, A. (2002) e-Encuestas Probabilísticas I. Los marcos. Estadística Española. Revista del Instituto Nacional de Estadística, 44 (151), 281-305.

Delors, J. (2001) La educación encierra un tesoro. Madrid, Santillana-UnESCO.

Demaziere, D.; Lessard, C. y Morrissetteet, J. (2013) Les effets de la Nouvelle Gestion Publique sur le travail des professionnels. Éducation et sociétés, 32, 5-20. 
DíAz, J.; Civís, M. y LongÁs, J. (2013) La gobernanza de redes socioeducativas: claves para una gestión exitosa. Teoría de la Educación, 25 (2), 213-230.

DíAz BECERRO, S. (2009) Introducción a las plataformas virtuales en la enseñanza. Temas para la Educación. Revista Digital para Profesionales de la Enseñanza, 2, 1-7.

Drucker, P. F. (1993) La sociedad postcapitalista. Barcelona, Ediciones Apóstrofe.

Embid Irujo, A. (2000) La enseñanza en España en el umbral del siglo XXI. Madrid, Tecnos. Eu (2013) Survey of Schools: ICT in Education. Bruxelles, European Union.

GIL, Ph. (2001) E-Formación. Bilbao, Ediciones Deusto.

Gimeno SACRISTÁN, J. (1994) La desregulación del currículum y la autonomía de los centros escolares. Signos. Teoría y Práctica de la Educación, 13, 4-20.

Gómez Llorente, L. (2000) Educación pública. Madrid, Morata.

Grupo Del Banco Mundial (2011) World Bank Group Education Strategy 2020: Learning for All: Investing in People's Knowledge and Skills to Promote Development. Consultado el 13 de mayo de 2014.

http://bit.ly/hTijbA

HARDT, M. y Negri, A. (2002) Imperio. Barcelona, Paidós.

INNERARITY, D. (2006) El nuevo espacio público. Madrid, Espasa.

LEwKowicz, I. (2012) Pensar sin Estado. La subjetividad en la era de la fluidez. Buenos Aires, Paidós.

Míguez, P. (2008) Las transformaciones recientes de los procesos de trabajo: desde la automatización hasta la revolución informática. Trabajo y sociedad, x (11), 1-20. Consultado el 2 de septiembre de 2014. http://goo.gl/Ewr7vT

MECD (2013) Datos y cifras. Curso escolar 2013/2014. Madrid, Secretaría General Técnica, Subdirección General de Documentación y Publicaciones. Consultado el 27 de febrero de 2014 . http://goo.gl/IGLloE

Mosquera, M. (2008) De la Etnografía antropológica a la Etnografía Virtual. Estudio de las relaciones sociales mediadas por Internet. Fermentum, 53, 532-549.

ODM (2014) Objetivos de Desarrollo del Milenio. Informe de 2013. Nueva York, Naciones Unidas.

PÉrez EsParrells, C. y Morales Sequera, S. (2012) La descentralización de la enseñanza no universitaria en España: análisis de convergencia desde la perspectiva del gasto. Presupuesto y Gasto Público, 67, 137-160.

Puelles Benítez, M. de (1993) Estudio teórico sobre las experiencias de descentralización educativa. Revista Iberoamericana de Educación, 3, 1-22.

Puelles Benítez, M. de (2011) La educación en el constitucionalismo español. Cuestiones Pedagógicas, 21, 15-35.

Stiglitz, J. E. (2003) Los felices 90. Madrid, Taurus.

TENTI FANFANI, E. (2010) El oficio del maestro en el siglo XXI, en VV. AA. La educación en el horizonte 2020. Madrid, Fundación Santillana. Consultado el 25 de julio de 2014. http://goo.gl/9i2VOv

Turkle, Sh. (1997) La vida en la pantalla. Barcelona, Paidós.

VARela Mallou, J. (dir.) (2008) El libro de texto ante la incorporación de las TIC a la enseñanza. Santiago de Compostela, CEDRO-UsC. Consultado el 26 de junio de 2014. http://goo.gl/rDTae. 
VERger, A. (2012) Globalización, reformas educativas y la nueva gestión del personal docente. Revista Docencia, 46, 1-13.

Verger, A. y Bonal, X. (2011) La estrategia educativa 2020 o las limitaciones del Banco Mundial para promover el "aprendizaje para todos". Educaçao \& Sociedade, 32 (117), 1-13.

VIÑao Frago, A. (1994) Sistemas educativos y espacios de poder: teorías, prácticas y usos de la descentralización en España. Revista Iberoamericana de Educación, 4, 29-64.

Walker, E. M. (2002) The Politics of School-Based Management: Understanding the Process of Devolving Authority in Urban School Districts. Education Policy Analysis Archives, 10 (33), 220-245.

\section{GLOSARIO}

CEIP-3: tercer caso de Centro de Educación Infantil y Primaria.

CEIP-4: cuarto caso de Centro de Educación Infantil y Primaria.

IES-2: segundo caso de Instituto de Educación Secundaria.

PM: profesor Matemáticas.

PP1: profesora de Educación Primaria (2. ${ }^{\circ}$.

PP2: profesora de Educación Primaria $\left(5^{\circ}\right)^{\circ}$.

P1CF: profesor/a Ciclo Formativo.

s1: secretaría de instituto. 